Commun. Fac. Sci. Univ. Ank. Ser. A1 Math. Stat.

Volume 68, Number 2, Pages 2258-2263(2019)

DOI: $10.31801 /$ cfsuasmas.476986

ISSN 1303-5991 E-ISSN 2618-6470

http://communications.science.ankara.edu.tr/index.php?series=A1

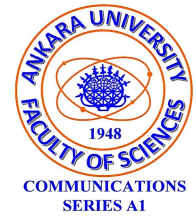

\title{
GENERALIZATIONS OF SOME RESULTS ON GENERALIZED
} POLYNOMIAL IDENTITIES

\author{
MÜNEVVER PINAR EROĞLU
}

\begin{abstract}
In this work, our aim is to generalize some of the results in 1 and [5. Precisely, we extend the result in [1] on commuting values of the same generalized derivations to the different generalized derivations case by a short proof. Also as an application, we extend a result in [5] on images of a linear map with derivations to generalized derivations case.
\end{abstract}

\section{INTRODUCTION}

Throughout, rings are always associative. Let $R$ be a ring. For $a, b \in R$, let $[a, b]=a b-b a$, the commutator of $a$ and $b$. For additive subgroups $A, B$ of $R$, let $[A, B]$ denote the additive subgroup of $R$ generated by all elements $[a, b]$ for $a \in A$ and $b \in B$. An additive map $\delta: R \rightarrow R$ is called a derivation if $\delta(x y)=\delta(x) y+x \delta(y)$ for all $x, y \in R$. Given $b \in R$, the map $x \mapsto b x-x b$ for $x \in R$ is called an inner derivation, denoted by $\operatorname{ad}(b)$, induced by the element $b$. A derivation of $R$ is called outer if it is not inner. An additive mapping $G: R \rightarrow R$ is called a generalized derivation of $R$ if there exists a derivation $\delta$ of $R$ such that $G(x y)=G(x) y+x \delta(y)$ for all $x, y \in R$. Evidently, any derivation is a generalized derivation. For $a, b \in R$, it is easy to see that the mapping $a x-x b$ is a generalized derivation of $R$ known as inner generalized derivation.

By a prime ring we mean a ring $R$ such that for $a, b \in R, a R b=0$ implies either $a=0$ or $b=0$. Throughout, $R$ is always a prime ring. Let $Q$ denote the maximal right ring of quotients of $R$. It is known that $Q$ is prime and its center, denoted by $C$, is a field, which is called the extended centroid of $R$. Let $Q{ }^{*} C C\left\{X_{1}, X_{2}, \cdots\right\}$ stand for the free product of the $C$-algebras $Q$ and $C\left\{X_{1}, X_{2}, \ldots, X_{n}, \ldots\right\}$, the free $C$-algebra in the noncommutative indeterminates $X_{1}, X_{2}, \cdots$. An element $\phi\left(x_{1}, \ldots, x_{n}\right)$ of the free product is called a generalized polynomial identity (gpi) on $R$, if $\phi\left(r_{1}, \ldots, r_{n}\right)=0$ for all $r_{1}, \ldots, r_{n} \in R$ (see [2] for more details).

Received by the editors: October 31, 2018; Accepted: January 23, 2019.

2010 Mathematics Subject Classification. 16N60, 16W25, 16R50.

Key words and phrases. Simple GPI-ring, prime ring, commutator ring, derivation, generalized derivation.

(C)2019 Ankara University Communications Faculty of Sciences University of Ankara-Series A1 Mathematics and Statistics 
Recently, most authors study some generalized polynomial identities on a prime ring and characterize the structure of maps involving in these identities (see [1], [3], [4] and [5]). In this way, they try to find out the structure of the ring. In this work, our aim is to generalize several of the works on generalized polynomial identities.

In [1], Ali et al. study a generalized polynomial identity with a commutator of the same generalized derivation. Precisely, they characterize the structure of a nonzero generalized derivation $G$ of $R$ such that $[G(u) u, G(v) v]=0$ for all $u, v \in f(R)$, the set of all evaluations in $R$ of the multilinear polynomial over $C$. In section 2 , we extend the result to the different generalized derivation case by a short proof, namely, we characterize the structure of two nonzero generalized derivations $G$ and $F$ of $R$ such that $[G(u) u, F(v) v]=0$ for all $u, v \in f(R)$ (see Theorem 2.1).

Motivated by the Noether-Skolem theorem, in [5] the author and T.-K. Lee characterize a linear differential map $\varphi(x)=\sum_{j} a_{j} \delta^{j}(x)$ for all $x \in R$ such that $\phi(R) \subseteq[R, R]$, where $R$ is a simple ring with a nonzero derivation $\delta$ and the $a_{j}$ 's are finitely many elements in $Q$. In section 3, as an application of the result, we consider the linear map for generalized derivations, namely, we characterize a linear map $\phi(x)=\sum_{j} a_{j} G^{j}(x)$ for all $x \in R$ such that $\phi(R) \subseteq[R, R]$, where $G$ is a generalized derivation of $R$ (see Theorem 3.5).

\section{A generalization of the Result in [1}

Throughout this section, $R$ is always a prime ring. Let $Q$ be the maximal right ring of quotients of $R$, and $C$ be its center. We will use the following notation for a multilinear polynomial over $C$ :

$$
f\left(X_{1}, \ldots, X_{n}\right)=\sum_{\sigma \in S_{n}} \alpha_{\sigma} X_{\sigma(1)} X_{\sigma(2)} \ldots X_{\sigma(n)}
$$

for some $\alpha_{\sigma} \in C$, and $S_{n}$ is the symmetric group of degree $n$. Let

$$
f(R):=\left\{f\left(r_{1}, \ldots, r_{n}\right) \mid r_{1} \ldots, r_{n} \in R\right\}
$$

the set of all evaluations in $R$ of the multilinear polynomial over $C$. We denote by $s_{4}$, the standard polynomial in four variables defined as follows:

$$
s_{4}\left(X_{1}, X_{2}, X_{3}, X_{4}\right)=\sum_{\sigma \in S_{4}}(-1)^{\sigma} X_{\sigma(1)} X_{\sigma(2)} X_{\sigma(3)} X_{\sigma(4)},
$$

where $(-1)^{\sigma}$ is the sign of a permutation $\sigma$ of the symmetric group of degree $4, S_{4}$.

In [1, Ali et al. proved that if $R$ is a prime ring of characteristic different 2, $f\left(X_{1}, \ldots, X_{n}\right)$ is a noncentral multilinear polynomial over $C$ and $G$ is a nonzero generalized derivation of $R$ such that

$$
\left[G\left(f\left(r_{1}, \ldots, r_{n}\right)\right) f\left(r_{1}, \ldots, r_{n}\right), G\left(f\left(s_{1}, \ldots, s_{n}\right)\right) f\left(s_{1}, \ldots, s_{n}\right)\right]=0
$$

for all $r_{1}, \ldots, r_{n}, s_{1}, \ldots, s_{n} \in R$, then there exists $c \in Q$ such that $G(x)=c x$ for all $x \in R$, moreover either $f\left(X_{1}, \ldots, X_{n}\right)^{2}$ is central or $R$ satisfies $s_{4}$. As an extension of the result, in this section we precisely prove the following. 
Theorem 2.1. Let $R$ be a prime ring of characteristic different 2 with extended centroid $C$ and $f\left(X_{1}, \ldots, X_{n}\right)$ a noncentral multilinear polynomial over $C$. Let $G$ and $F$ be nonzero generalized derivations of $R$ such that

$$
\left[G\left(f\left(r_{1}, \ldots, r_{n}\right)\right) f\left(r_{1}, \ldots, r_{n}\right), F\left(f\left(s_{1}, \ldots, s_{n}\right)\right) f\left(s_{1}, \ldots, s_{n}\right)\right]=0
$$

for all $r_{1}, \ldots, r_{n}, s_{1}, \ldots, s_{n} \in R$. Then $f\left(X_{1}, \ldots, X_{n}\right)^{2}$ is central valued on $R$ and moreover, one of the following statements holds:

(i) There exists $\lambda \in C$ such that $G(x)=\lambda x$ for all $x \in R$;

(ii) There exists $\mu \in C$ such that $F(x)=\mu x$ for all $x \in R$;

(iii) There exist $a, c \in Q$ such that $G(x)=a x$ and $F(x)=c x$ for all $x \in R$ and $[a, c]=0$.

Proof. Suppose first $G\left(f\left(r_{1}, \ldots, r_{n}\right)\right) f\left(r_{1}, \ldots, r_{n}\right) \in C$ for all $r_{1}, \ldots, r_{n} \in R$. In view of Lemma 3 in [4], $f\left(X_{1}, \ldots, X_{n}\right)^{2}$ is central valued on $R$ and also there exists $\lambda \in C$ such that $G(x)=\lambda x$ for all $x \in R$, as desired for $(i)$. By the same arguments, if $F\left(f\left(s_{1}, \ldots, s_{n}\right)\right) f\left(s_{1}, \ldots, s_{n}\right) \in C$ for all $s_{1}, \ldots, s_{n} \in R$, then $f\left(X_{1}, \ldots, X_{n}\right)^{2}$ is central valued on $R$ and also there exists $\mu \in C$ such that $F(x)=\mu x$ for all $x \in R$, as desired for $(i i)$. Therefore we may assume that there exist $s_{1}, \ldots, s_{n} \in R$ such that $v:=F\left(f\left(s_{1}, \ldots, s_{n}\right)\right) f\left(s_{1}, \ldots, s_{n}\right) \notin C$. Then by (1),

$$
\left[G\left(f\left(r_{1}, \ldots, r_{n}\right)\right) f\left(r_{1}, \ldots, r_{n}\right), v\right]=0
$$

for all $r_{1}, \ldots, r_{n} \in R$. Let $\delta$ be an inner derivation induced by $v \in R$, i.e., $\delta(x)=$ $[x, v]$ for all $x \in R$. So $v \notin C$ implies $\delta \neq 0$. It follows from (2) that

$$
\delta\left(G\left(f\left(r_{1}, \ldots, r_{n}\right)\right) f\left(r_{1}, \ldots, r_{n}\right)\right)=0
$$

for all $r_{1}, \ldots, r_{n} \in R$. In view of $\left[\underline{3}, f\left(X_{1}, \ldots, X_{n}\right)^{2}\right.$ is central valued on $R$ and moreover, there exists $a \in Q$ such that $G(x)=a x$ for all $x \in R$ and $\delta(a)=0$. Then $[a, v]=0$. It means

$$
\left[a, F\left(f\left(s_{1}, \ldots, s_{n}\right)\right) f\left(s_{1}, \ldots, s_{n}\right)\right]=0
$$

for all $s_{1}, \ldots, s_{n} \in R$ with $F\left(f\left(s_{1}, \ldots, s_{n}\right)\right) f\left(s_{1}, \ldots, s_{n}\right) \notin C$. However, (3) holds for all $s_{1}, \ldots, s_{n} \in R$ with $F\left(f\left(s_{1}, \ldots, s_{n}\right)\right) f\left(s_{1}, \ldots, s_{n}\right) \in C$. Thus, (3) holds for all $s_{1}, \ldots, s_{n} \in R$. Then by the same arguments above for $(2)$, we have $f\left(X_{1}, \ldots, X_{n}\right)^{2}$ is central valued on $R$ and moreover, there exists $c \in Q$ such that $F(x)=c x$ for all $x \in R$ and $[a, c]=0$ in view of [3]. It means there exist $a, c \in Q$ such that $G(x)=a x$ and $F(x)=c x$ for all $x \in R$ and $[a, c]=0$, as desired for (iii). This completes the proof.

The main theorem of [1] is then an immediate consequence of Theorem 2.1. But the following is a sharper characterization. 
Corollary 2.2. Let $R$ be a prime ring of characteristic different 2 with extended centroid $C$ and $f\left(X_{1}, \ldots, X_{n}\right)$ a noncentral multilinear polynomial over $C$. Let $G$ be a nonzero generalized derivation of $R$ such that

$$
\left[G\left(f\left(r_{1}, \ldots, r_{n}\right)\right) f\left(r_{1}, \ldots, r_{n}\right), G\left(f\left(s_{1}, \ldots, s_{n}\right)\right) f\left(s_{1}, \ldots, s_{n}\right)\right]=0
$$

for all $r_{1}, \ldots, r_{n}, s_{1}, \ldots, s_{n} \in R$. Then $f\left(X_{1}, \ldots, X_{n}\right)^{2}$ is central valued on $R$ and moreover, there exists $a \in Q$ such that $G(x)=$ ax for all $x \in R$.

\section{An application of a Result in 5 ]}

Throughout this section, $R$ is always a prime ring. Let $Q$ be the maximal right ring of quotients of $R$, and $C$ be its center. It is known that any derivation $\delta: R \rightarrow R$ can be uniquely extended to a derivation of $Q$, denoted by $\delta$ also. A derivation $\delta: R \rightarrow R$ is called $\mathrm{X}$-inner if its extension to $Q$ is inner; that is, $\delta=\operatorname{ad}(b)$ for some $b \in Q$. Otherwise, it is called X-outer. In [8, Theorem 4], T.-K. Lee showed that a generalized derivation $G$ of a prime ring $R$ is of form $G(x)=a x+\delta(x)$ for some $a \in Q$ and a derivation $\delta$ of $R$. Moreover $a \in Q$ and $\delta$ are uniquely determined by $G$. Also $\delta$ is called the associated derivation of $G$. A generalized derivation $G$ is called X-inner if its associated derivation is $\mathrm{X}$-inner; otherwise it is called $\mathrm{X}$-outer. Following [5], let

$$
Q[t ; \delta]:=\left\{a_{0}+a_{1} t+\cdots+a_{n} t^{n} \mid a_{0}, \ldots, a_{n} \in Q, n \geq 0\right\},
$$

be the Ore extension of $Q$ by $\delta$ endowed with the multiplication rule: $t x=x t+\delta(x)$ for $x \in Q$. A polynomial $f(t)=a_{0}+a_{1} t+\cdots+a_{n} t^{n} \in Q[t ; \delta]$ has degree $n$ if $a_{n} \neq 0$, denoted by $\operatorname{deg} f(t)=n$, and is called monic if $a_{n}=1$.

Given $f(t)=a_{0}+a_{1} t+\cdots+a_{n} t^{n} \in Q[t ; \delta]$ and a derivation $\delta$ of $R$, we define $f(\delta)=\left(a_{0}\right)_{L} \operatorname{id}_{R}+\left(a_{1}\right)_{L} \delta+\cdots+\left(a_{n}\right)_{L} \delta^{n}$, where $\operatorname{id}_{R}$ is the identity map of $R$.

Definition 3.1. A derivation $\delta: R \rightarrow R$ is said to be quasi-algebraic if there exist $b_{1}, \ldots, b_{n-1}, b \in Q$ such that for all $x \in R$,

$$
\delta^{n}(x)+b_{1} \delta^{n-1}(x)+\cdots+b_{n-1} \delta(x)=b x-x b .
$$

The least integer $n$ is called the quasi-algebraic degree or the outer degree of the derivation $\delta$ and is denoted by out $-\operatorname{deg}(\delta)$. Clearly, out $-\operatorname{deg}(\delta)=1$ if and only if $\delta$ is $X$-inner. We also let out- $\operatorname{deg}(\delta)=\infty$ if $\delta$ is not quasi-algebraic.

Remark 3.2. Let $\delta: R \rightarrow R$ be a quasi-algebraic derivation. We apply Kharchenko's theorem [6, Corollaries 2 and 3] (see also [7, Theorem 2]). If $\operatorname{char} R=0$, then $\delta=\operatorname{ad}(b)$ for some $b \in Q$. If char $R=p>0$, then $\delta, \delta^{p}, \delta^{p^{2}}, \ldots$ are linearly dependent over $C$ modulo inner derivations of $Q$. Let $s \geq 0$ be the minimal integer such that

$$
\delta^{p^{s}}, \delta^{p^{s-1}}, \cdots, \delta^{p}, \delta
$$


are linearly dependent over $C$ modulo inner derivations of $Q$. By the minimality of $s$, there exist $\alpha_{i} \in C$ and $b \in Q$ such that

$$
\delta^{p^{s}}+\alpha_{1} \delta^{p^{s-1}}+\cdots+\alpha_{s} \delta=a d(b) .
$$

By the minimality of $s$ again, it is easy to see that $\delta\left(\alpha_{i}\right)=0$ and $\delta(b) \in C$. In this case, we have out $-\operatorname{deg}(\delta)=p^{s}$.

Definition 3.3. Let $\delta$ be a quasi-algebraic derivation of $R$. We define $p(t):=t$ if out $-\operatorname{deg}(\delta)=1$ and $p(t):=t^{p^{s}}+\alpha_{1} t^{p^{s-1}}+\cdots+\alpha_{s} t$ if char $R=p>0$. In either case, $p(t)$ is called the associated polynomial of $\delta$. Note that $p(\delta)=$ ad $(b)$ for some $b \in Q$ and $p(t) \in C^{(\delta)}[t ; \delta] \subseteq Q[t ; \delta]$, where $C^{(\delta)}:=\{\beta \in C \mid \delta(\beta)=0\}$, the subfield of constants of $\delta$ in $C$. Note that $C^{(\delta)}[t ; \delta]=C^{(\delta)}[t]$.

In [5, Theorem 1.5], the author and T.-K. Lee characterize a linear differential map in the following result.

Theorem 3.4. Let $R$ be a simple GPI-ring with a nonzero derivation $\delta$. Then, for a nonzero polynomial $f(t) \in Q[t ; \delta], f(\delta)(R) \subseteq[R, R]$ if and only if $\delta$ is quasialgebraic and $p(t) \mid f(t)$, where $p(t)$ is the associated polynomial of $\delta$.

In this section our main is to give an application of the above result to generalization derivations.

Theorem 3.5. Let $R$ be a simple GPI-ring, $b_{0}, \ldots, b_{m} \in Q$ with $b_{0} \neq 0$ and $G$ a nonzero generalized derivation of $R$. Assume that

$$
\sum_{i=0}^{m} b_{i} G^{i}(x) \in[R, R]
$$

for all $x \in R$. If $G$ is $X$-outer then there exist $b, c \in Q$ and $\alpha_{1}, \cdots, \alpha_{s} \in C$ such that

$$
G^{p^{s}}+\alpha_{1} G^{p^{s-1}}+\cdots+\alpha_{s} G=c x-x b
$$

for all $x \in R$.

Proof. In view of [8, Theorem 4], there exist $a \in Q$ and a derivation $\delta$ of $R$ such that $G(x)=a x+\delta(x)$ for all $x \in R$. Thus by a direct computation, it is easy to see that $\sum_{i=0}^{m} b_{i} G^{i}(x)=\sum_{i=0}^{m} w_{i} \delta^{i}(x)$ for some $w_{i} \in Q$. Denote $f(t)=\sum_{i=0}^{m} w_{i} t^{i}(x) \in$ $Q[t ; \delta]$. So by our hypothesis, we have $f(\delta)(R) \subseteq[R, R]$. It follows from Theorem 3.4 that $\delta$ is a quasi-algebraic. Therefore by Remark (3.2), since $G$ is X-outer we may assume that $\operatorname{char}(R)=p$, and so there exist $\alpha_{i} \in C$ and $b \in Q$ such that

$$
\delta^{p^{s}}+\alpha_{1} \delta^{p^{s-1}}+\cdots+\alpha_{s} \delta=\operatorname{ad}(b) .
$$


On the other hand, since $\operatorname{char}(R)=p>0$ there exist $c_{1}, c_{2}, \cdots, c_{s} \in Q$ such that

$$
G^{p}(x)=c_{1} x+\delta^{p}(x), G^{p^{2}}(x)=c_{2} x+\delta^{p^{2}}(x), \cdots, G^{p^{s}}(x)=c_{s} x+\delta^{p^{s}}(x)
$$

for all $x \in R$. In view of (4), we obtain

$c_{s} x+\delta^{p^{s}}(x)+\alpha_{1}\left(c_{s-1} x+\delta^{s^{s-1}}(x)\right)+\cdots+\alpha_{s}(a x+\delta(x))=[b, x]+\left(c_{s}+\alpha_{1} c_{s-1}+\cdots+\alpha_{s} a\right) x$

for all $x \in R$. It means

$G^{p^{s}(x)}+\alpha_{1} G^{p^{s-1}}(x)+\cdots+\alpha_{s} G(x)=b x-x b+\left(c_{s}+\alpha_{1} c_{s-1}+\cdots+\alpha_{s-1} c_{1}+\alpha_{s} a\right) x$

and so

$$
G^{p^{s}}+\alpha_{1} G^{p^{s-1}}+\cdots+\alpha_{s} G=(b+w) x-x b
$$

for all $x \in R$, where $w=c_{s}+\alpha_{1} c_{s-1}+\cdots+\alpha_{s} a \in Q$, as desired. This completes the proof.

\section{REFERENCES}

[1] Ali A., De Filippis V. and Shujat F., Commuting values of generalized derivations on multilinear polynomials, Comm. Algebra 42, (2014), 3699-3707.

[2] Beidar K.I., Martindale W.S. and Mikhalev A.V., Rings with generalized identities, Pure and Aplied Math., Dekker, New York, 1996.

[3] Carini L. and De Filippis V., Centralizers of generalized derivations on multilinear polynomials in prime rings, Siberian Math. Journal 53(6), (2012) 1051-1060.

[4] Demir Ç. and Argaç N., Prime rings with generalized derivations on right ideals, Algebra Colloq. 18, (2011), 987-998.

[5] Eroğlu M.P. and Lee T.-K., On the images of polynomials of derivations, Comm. Algebra 45(10), (2017), 4550-4556.

[6] Kharchenko V.K., Differential identities of prime rings, Algebra i Logika 17, (1978), 220-238. (Engl. Transl., Algebra and Logic 17, (1978), 154-168.)

[7] Kharchenko V.K., Differential identities of semiprime rings, Algebra i Logika 18, (1979), 86119. (Engl. Transl., Algebra and Logic 18, (1979), 58-80.)

[8] Lee T.-K., Generalized derivations of left faithful rings, Comm. Algebra 27, (1999), 4057-4073. Current address: Department of Mathematics, Science Faculty Dokuz Eylül University Izmir Turkey

E-mail address: mpinar.eroglu@deu.edu.tr

ORCID Address: http://orcid.org/0000-0002-7523-9812 